\title{
Atomic-Scale Characterization of II-VI Compound Semiconductors
}

\author{
DAVID J. SMITH ${ }^{1,2}$ \\ 1.-Department of Physics, Arizona State University, Tempe, AZ 85287, USA. 2.-e-mail: david. \\ smith@asu.edu
}

\begin{abstract}
Alloys of II-VI compound semiconductors with suitable band gap selection potentially provide broad coverage of wavelengths for photodetector applications. Achievement of high-quality epitaxial growth is, however, essential for successful development of integrated photonic and optoelectronic devices. Atomic-scale characterization of structural defects in II-VI heterostructures using electron microscopy plays an invaluable role in accomplishing this goal. This paper reviews some recent high-resolution studies of II-VI compound semiconductors with zincblende crystal structure, as grown epitaxially on commonly used substrates. Exploratory studies using aberration-corrected electron microscopes are also briefly considered.
\end{abstract}

Key words: High-resolution electron microscopy, zinc telluride, cadmium telluride, mercury cadmium telluride, mercury cadmium selenide, aberration-corrected electron microscopy

\section{INTRODUCTION}

Ternary (and quaternary) alloys of II-VI compound semiconductors containing appropriate combinations of $(\mathrm{HgCdZnMg})(\mathrm{SeTe})$ can, in principle, provide a selection of band gaps that range over the spectrum of wavelengths from near-ultraviolet to far-infrared (IR) (Fig. 1). Hence, there is much ongoing interest in and motivation for further development of optoelectronic devices as well as detectors based on these materials; For example, IR detectors using mercury cadmium telluride (MCT) continue to be of central importance for sensing applications. ${ }^{1}$ Thus, epitaxial growth of MCT on large-area, low-cost substrates, rather than bulk, lattice-matched cadmium zinc telluride, is a highly active field of current research. ${ }^{2,3}$ Similarly, CdTe (band gap $1.44 \mathrm{eV}$ ) and $\mathrm{ZnTe}$ (band gap $2.26 \mathrm{eV}$ ) represent potentially viable candidates for photodetectors or multijunction solar cells, but highquality crystalline material is considered to be essential to avoid degradation in device performance. Developing a successful strategy for defect

(Received April 18, 2013; accepted July 9, 2013;

published online August 14, 2013) reduction during epitaxial growth of these and other II-VI materials, given the likely lattice mismatch with any potential substrate and inevitable differences in their thermal expansion coefficients, becomes a challenging task.

Characterization of defects is critical in the quest for growth of compound semiconductors of the highest possible quality. Examination by electron microscopy provides imaging, diffraction, and analytical signals that give complementary information about local microstructure, crystallography, and chemical composition. Feedback from the electron microscopist about the nature and type of any structural defects that are present then enables crystal growers and device engineers to optimize growth conditions and eventually to improve device performance. The spatial resolution of transmission electron microscopy (TEM) has steadily improved over the years, and observations at atomic level have been made for many types of material and different structural defects. ${ }^{4}$ The purpose of this article is to provide a brief overview of some recent atomic-level electron microscopy studies that illustrate epitaxial growth of II-VI compound semiconductors on several common substrates. Opportunities for closer examination of these materials with the latest 


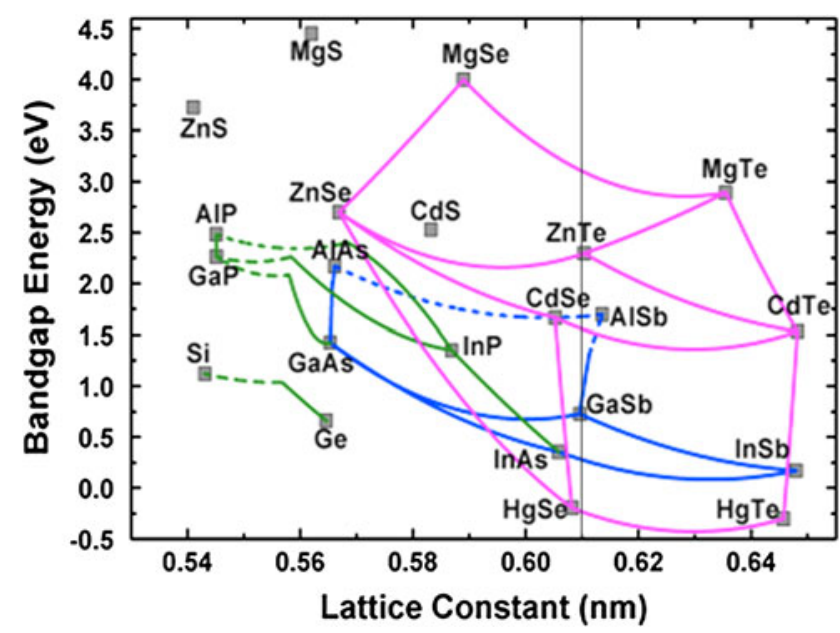

Fig. 1. (Color online) Calculated band gaps of various II-VI and III-V compound semiconductors versus lattice parameter. The vertical line indicates lattice constant of $6.1 \AA$

generation of aberration-corrected electron microscopes (ACEMs) are also discussed.

\section{BACKGROUND}

Many of the common II-VI compound semiconductors have zincblende crystal structure, with lattice parameters ranging from $0.567 \mathrm{~nm}(\mathrm{ZnSe})$ to $0.648 \mathrm{~nm}(\mathrm{CdTe})$. Lattice-fringe imaging of these materials in the major $<110>,<100>$, and $<112>$ projections using TEM is relatively straightforward with modern instruments. The $<110>$ projection is most often used for examination of semiconductor interfaces and growth defects, and crystal polarity can usually be determined by studying the characteristic image appearance at certain thicknesses and objective-lens defocus settings. ${ }^{5}$ However, individual atomic columns, often referred to as "dumbbells," cannot be separately resolved when imaging in this projection unless $\{004\}$-type reflections contribute to the image formation process. This performance requirement is quite demanding and could initially only be achieved by dedicated high-voltage, high-resolution electron microscopes. ${ }^{7}$ ACEMs, which are capable of pushing information limits to beyond the 1.0-A level, have since become available, so that projected atomic columns can be resolved in all zincblende compound semiconductors for observation in this viewing direction. ${ }^{8}$

Specimen damage during the process of ion milling is well known to be a problematic issue when preparing cross-sections of II-VI semiconductors for TEM observation, and can easily lead to erroneous interpretation of defect microstructure ${ }^{9}$ For example, argon-ion milling was shown to cause $\{111\}$-type stacking-fault dislocation loops, which could however be effectively eliminated by instead using reactive iodine milling (albeit also accompanied by greatly increased damage to components of the vacuum system). ${ }^{10}$ In the case of HgCdTe alloys, it was shown that the amount of ion-induced damage could be markedly reduced by milling at low glancing angles and with lower-energy $(<2 \mathrm{keV})$ ion beams, and with the sample cooled to liquid-nitrogen temperature. ${ }^{11}$ This approach has since proven to be effective for thinning other II-VI semiconductors, and should be adopted as standard practice for this type of material. Wedge polishing is an alternative thinning method that could be used to ameliorate ion-damage effects, although, in our experience, sample brittleness and low mechanical strength mitigate strongly against successful thinning of II-VI samples to electron transparency. Chemical polishing is yet another possibility for sample thinning, but one that does not seem to have been explored in the specific context of achieving extensive areas of II-VI semiconductor materials which are thin enough for TEM examination. Moreover, this approach may not be viable when preparing a heterostructure consisting of two or more different materials because of inherent differences in thinning rates during etching. Finally, it should be appreciated that, despite the effectiveness of focused-ion-beam milling for site-specific semiconductor device applications, this technique seems unsuited for routine thinning of II-VI zincblende semiconductors, at least for high-resolution observations, because of the inherent susceptibility of their surfaces to ion-induced damage.

\section{RESULTS AND DISCUSSION}

Characterization of II-VI compound semiconductors by TEM methods has been widely reported in the scientific literature, with atomic-resolution studies becoming more commonplace as instrumental performance has progressively improved. The examples described in the following paragraphs are illustrative of these developments. Further information about substrate preparation as well as material-specific growth and annealing conditions can be found in the cited references.

\section{ZnTe}

\section{Si(100) and Si(211) Substrates}

Substrates based on silicon are of much interest for monolithic integration of photonic materials, but high defect densities due to lattice mismatch have historically represented a major limitation on device performance. Thus, much effort has been directed towards composite substrates that serve to relieve the inevitable strain caused by the large differences in lattice parameter. Growth of very thick $(\sim 5 \mu \mathrm{m}$ to $9 \mu \mathrm{m}) \mathrm{ZnTe}$ epilayers on $\mathrm{Si}(100)$ and $\mathrm{Si}(211)$ substrates has recently received renewed attention to provide possible composite substrates for subsequent $\mathrm{HgCdTe} / \mathrm{CdTe}$ growth. ${ }^{3}$ Experiments with different $\mathrm{Zn} / \mathrm{Te}$ flux ratios showed that $\mathrm{ZnTe}(211) /$ $\mathrm{Si}(211)$ heterostructures grown under Te-rich or equal $\mathrm{Zn}$ and Te fluxes had much better crystal 
quality than those grown under Zn-rich conditions, as measured by full-width at half-maximum of x-ray diffraction (XRD) spectra, and also using etch-pitdensity measurements. In contrast, the opposite trend was observed to be the case for $\mathrm{ZnTe}(100) /$ $\mathrm{Si}(100)$ structures, with better quality being obtained for material grown in a Zn-rich environment. Figure 2a shows a high-resolution electron micrograph of a typical cross-section of a $\mathrm{ZnTe}(211) /$ $\mathrm{Si}(211)$ interface. No defects are visible in this region of the interface, although there is a slight rotation, measured to be $\sim 2.3^{\circ}$, between the vertical $\{111\}$-type lattice fringes in the two materials. Figure $2 \mathrm{~b}$ shows the same region of the interface after inverse Fourier filtering was applied to the original micrograph. The filtered image reveals a pseudoperiodic array of interfacial misfit dislocations, indicated by the arrows. Measurements show that the average separation between these dislocations corresponds closely to the value expected for $\mathrm{ZnTe} / \mathrm{Si}$ lattice mismatch, meaning that the interface itself must be close to fully relaxed.

\section{GaSb, InAs, InP, and GaAs (100) Substrates}

Growth of thick ZnTe(100) epilayers on common $\{100\}$-oriented III-V substrates has been investigated as a possible alternative approach to achieving low-cost virtual substrates for optoelectronic applications such as photodetectors, light-emitting diodes, and multijunction solar cells. ${ }^{12,13}$ Substrates that have been studied include GaSb $(0.13 \%$ mismatch with $\mathrm{ZnTe}), \operatorname{InAs}(0.75 \%), \operatorname{InP}(3.85 \%)$, and GaAs (7.30\%). All of these ZnTe samples $(\sim 2.4 \mu \mathrm{m}$ thick) were grown by molecular beam epitaxy (MBE) under virtually identical conditions, so any differences in materials properties and microstructure can be attributed to interfacial strain caused primarily by lattice-mismatch differences, as well as thermal expansion coefficients and growth temperature. XRD was used to analyze the residual strain in the $\mathrm{ZnTe}$ epilayer, photoluminescence (PL) was used to determine the optical properties, and TEM was used to characterize any differences in microstructure. In the case of the highly coherent $\mathrm{ZnTe}(100) / \mathrm{GaSb}(100)$ heterostructures, very wellseparated interfacial dislocations were occasionally visible, but, as shown in Fig. 3a, it was otherwise very difficult to determine the exact position of the interface because the two separate layers have virtually the same average atomic number. ${ }^{14}$ Progressively more interfacial defects became visible as the epilayer-substrate mismatch was increased. Standard Burgers' circuit analysis was used to identify both perfect $60^{\circ}$ dislocations, as well as Lomer edge dislocations, at the $\operatorname{ZnTe}(100) / \operatorname{InP}(100)$ and $\mathrm{ZnTe}(100) / \mathrm{GaAs}(100)$ interfaces. ${ }^{13}$ Figure $3 \mathrm{~b}$ shows a highly magnified image of the latter heterointerface, showing two closely separated $60^{\circ}$ dislocations and four Lomer edge dislocations. Analysis of the defect types and separation along the interface was
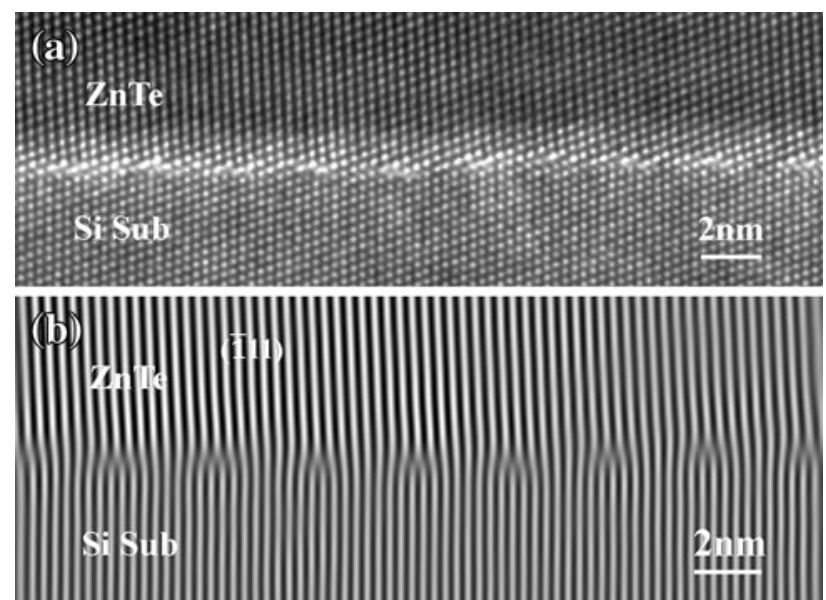

Fig. 2. (a) High-resolution cross-section image of $\mathrm{ZnTe}(211) / \mathrm{Si}(211)$ interface, also showing slight rotation $\left(\sim 2.3^{\circ}\right)$ between vertical $\{111\}$ type lattice fringes; (b) Corresponding Fourier-filtered image revealing interfacial misfit dislocations. ${ }^{3}$
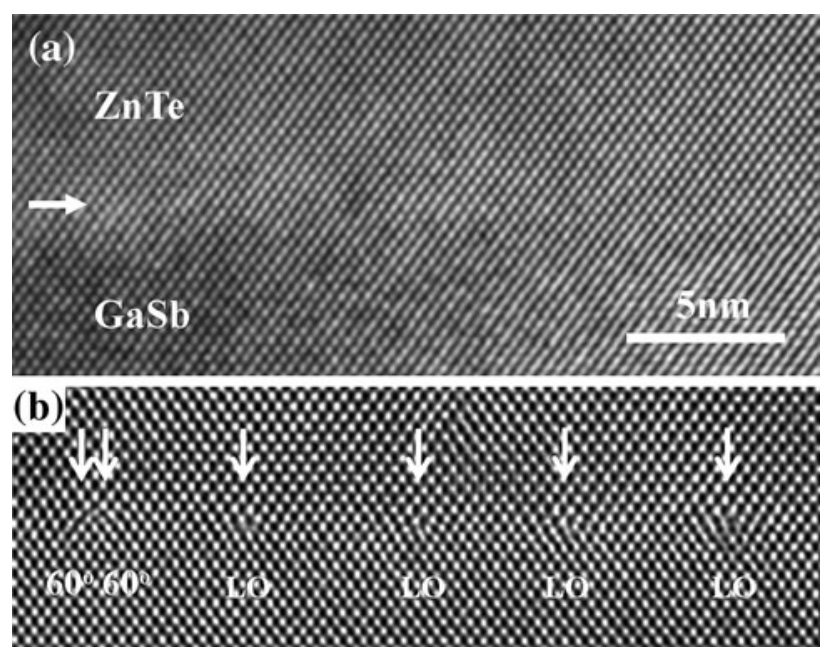

Fig. 3. (a) High-resolution electron micrograph showing highly coherent $\mathrm{ZnTe} / \mathrm{GaSb}(100)$ interface; ${ }^{14}$ (b) high-resolution electron micrograph of $\mathrm{ZnTe} / \mathrm{GaAs}(100)$ interface identifying Lomer edge (LO) and $60^{\circ}$ perfect dislocations. ${ }^{13}$

then used to estimate the residual interfacial strain, which was found to be $\sim 0.01 \%$ for the $\mathrm{ZnTe} / \mathrm{InP}$ sample and $\sim 0.09 \%$ for the $\mathrm{ZnTe} / \mathrm{GaAs}$ sample. ${ }^{13}$ Thus, these interfaces could be regarded as being completely relaxed to within experimental error.

\section{CdTe}

\section{Si(100) Substrate}

Composite $\mathrm{CdTe} / \mathrm{Si}$ substrates for subsequent growth of $\mathrm{HgCdTe}$ potentially offer several advantages over conventional CdTe bulk substrates or lattice-matched CdZnTe substrates, including good uniformity over large areas and mechanical strength. Conversely, the large lattice-constant mismatch between CdTe and $\mathrm{Si}$ represents a 
considerable barrier to successful heteroepitaxial growth. High-resolution observations of $\mathrm{CdTe} /$ $\mathrm{Si}(100)$ heterostructures initially revealed extensive \{111\}-type lamellar twinning as well as doubledomain CdTe growth, which was attributed to the rotational symmetry of the clean, reconstructed $\operatorname{Si}(100)$ surface. ${ }^{15}$ Large miscuts $\left(\sim 4^{\circ}\right.$ to $\left.8^{\circ}\right)$ to the $\mathrm{Si}(100)$ substrate surface were used to lower the growth symmetry and to reduce the relative widths of step terraces. However, the improvements in structural quality achieved were not really substantial until slight rotations of the surface offcut were also introduced. ${ }^{16}$ Buffer layers of Ge, which has a lattice parameter intermediate between those of CdTe and $\mathrm{Si}$, were also briefly investigated during this study of $\mathrm{Si}(100)$ substrates. High-resolution studies (not shown here) revealed that an array of pseudoperiodic $\mathbf{a} / 2<110>$ Lomer edge dislocations accommodated most of the lattice misfit at the $\operatorname{CdTe}(100) / \mathrm{Ge}(100)$ interface. $^{17}$

\section{ZnTe(111)/Si(111) Substrates}

During further experiments with $\mathrm{Si}(111)$ substrates, thin $(\sim 5 \mathrm{~nm}) \mathrm{ZnTe}$ buffer layers were introduced to mediate the large lattice mismatch and epitaxial strain between the CdTe epilayer and the Si substrate. ${ }^{18}$ Electron diffraction patterns indicated excellent alignment between the diffraction spots of the three materials, while small-probe microanalysis showed some limited $\mathrm{Cd}$ and $\mathrm{Zn}$ interdiffusion between the CdTe and ZnTe layers, which was attributed to the extensive annealing treatments applied during growth. High-resolution images of the $\mathrm{ZnTe}(111) / \mathrm{Si}(111)$ interface, as shown in Fig. 4, confirmed the abrupt nature of the interface, and also demonstrated the close alignment of lattice planes in these two materials.

\section{ZnTe(211)/Si(211) Substrate}

The CdTe(211)B surface, ideally composed of (111) terraces and (100) steps, is the preferred orientation choice for $\mathrm{HgCdTe}$ epitaxial growth because reduced twinning and less antiphase domain formation are expected. However, the lattice parameter mismatch of $\sim 18 \%$ between CdTe and $\mathrm{Si}$ remains an important consideration. Thus, thin $(\sim 2 \mathrm{~nm}$ to $10 \mathrm{~nm})$ ZnTe buffer layers were again grown first to alleviate the mismatch strain between the materials, and also to preserve the (211) substrate orientation. ${ }^{19}$ Small-probe microanalysis showed strong evidence for considerable $\mathrm{Zn}$ diffusion away from the substrate, which was presumed to have occurred during CdTe deposition and sample annealing. ${ }^{15}$ High-resolution images of the (Cd,Zn)Te(211)/Si(211) heterointerface showed many twinning defects that terminated close to the substrate, as well as small rotation angles between the lattice-fringe directions upon crossing the plane of the interface. Later growth strategies involved different approaches to substrate preparation as

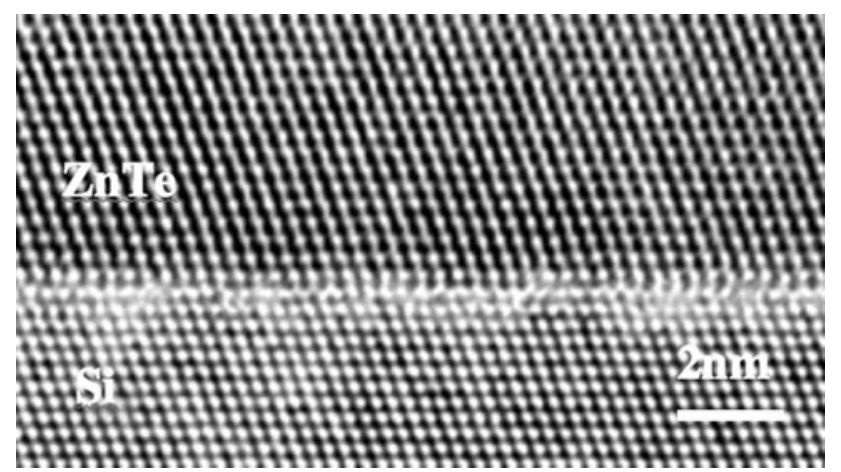

Fig. 4. Highly enlarged image of $\mathrm{ZnTe}(111) / \mathrm{Si}(111)$ interface showing close alignment of lattice fringes of the two materials. ${ }^{15}$

well as periodic annealing cycles that were intended to enhance dislocation interactions and annihilation. $^{20}$ High-resolution electron micrographs ${ }^{21}$ of the $\mathrm{CdTe}(211) \mathrm{B} / \mathrm{ZnTe} / \mathrm{Si}$ interface region, such as that shown in Fig. 5, reveal many short, inclined \{111\}-type stacking faults and twinning defects, while electron diffraction patterns (inset) show slight rotation angles $\left(\sim 3.5^{\circ}\right)$ between the $\mathrm{CdTe} / \mathrm{Si}$ lattice planes, which is similar to the example of the $\mathrm{ZnTe}(211) / \mathrm{Si}(211)$ heterostructure shown in Fig. 2a.

\section{CdSe/CdTe Superlattice}

\section{ZnTe(100)/GaSb(100) Substrate}

Composite substrates consisting of closely latticematched $\mathrm{ZnTe} / \mathrm{GaSb}$ represent an attractive option for growth of other II-VI materials. In particular, superlattices (SLs) consisting of $\mathrm{CdSe} / \mathrm{CdTe}$ are potentially of interest for solar-cell applications since band-gap engineering can be simply accomplished by adjusting the SL layer thicknesses. ${ }^{22}$ Thus, several CdSe/CdTe SLs were grown on composite $\mathrm{ZnTe}(100) / \mathrm{GaSb}(100)$ substrates, and the corresponding optical and structural properties were extensively investigated using PL, XRD, and TEM techniques. ${ }^{22}$ Low-magnification images demonstrated overall high structural quality, while satellite diffraction spots visible in selected-area electron diffraction patterns confirmed the excellent uniformity of the SL layer thicknesses. High-resolution lattice images, such as that shown in Fig. 6, also demonstrated the SL crystallinity at the atomic scale.

\section{HgCdTe}

\section{CdZnTe(211)B Substrate}

Bulk CdZnTe has historically been the substrate of choice for epitaxial growth of $\mathrm{HgCdTe}$ IR detector materials, mostly because of lattice-matching and chemical compatibility, even though there are obvious disadvantages to using CdZnTe such as production cost, limited wafer size, and material inhomogeneities. Electron microscopy studies have 


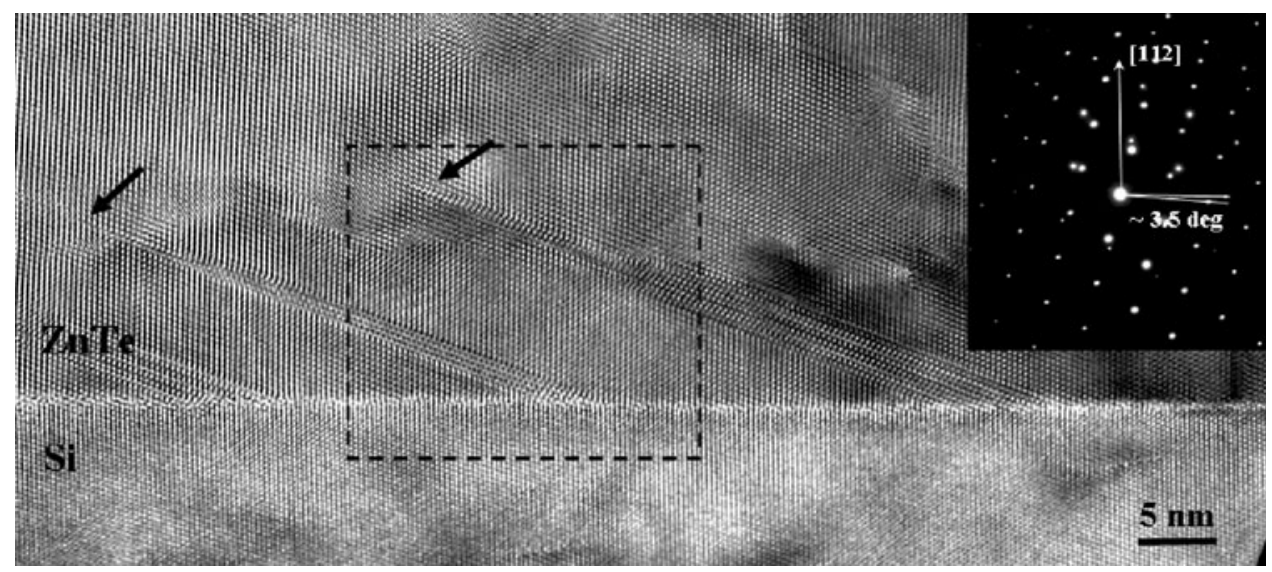

Fig. 5. Cross-sectional electron micrograph showing region of $\mathrm{CdTe}(211) / \mathrm{ZnTe}(211) / \mathrm{Si}(211)$ interface with short $\{111\}$-type stacking and twinning defects originating at the Si substrate surface. Inset electron diffraction pattern illustrates slight rotation $\left(\sim 3.5^{\circ}\right)$ between $\mathrm{CdTe} / \mathrm{Si}$ lattice planes. $^{21}$

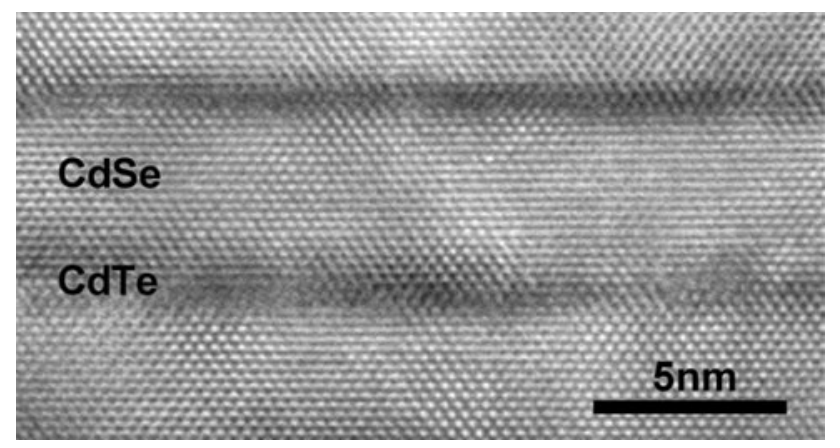

Fig. 6. High-resolution lattice image of $\mathrm{CdSe} / \mathrm{CdTe} \mathrm{SL}$ showing excellent crystallinity. ${ }^{22}$

featured prominently in the pursuit of higherquality MBE-grown material via attempts to optimize the HgCdTe growth conditions, especially the growth temperature window. The occurrence of $\mathrm{HgCdTe}$ surface crater defects was shown to be associated with the development of polycrystalline morphology, and high-resolution observations revealed finely spaced $\mathrm{HgCdTe} / \mathrm{Te}$ intergrowths that had nucleated within the $\mathrm{HgCdTe}$ epilayers. ${ }^{23}$ $\mathrm{SLs}$ of $\mathrm{HgTe} / \mathrm{Hg}_{0.05} \mathrm{Cd}_{0.95} \mathrm{Te}$ grown on $\mathrm{CdZnTe}(211)$ substrates have also been studied, and high-resolution studies using annular dark-field (or Z-contrast) imaging revealed interdiffusion and changes in well/barrier widths as a result of low-temperature annealing. ${ }^{24}$

\section{CdTe/GaAs(211)B Substrate}

Experiments with substrates other than CdZnTe for epitaxial growth of $\mathrm{HgCdTe}$ alloys are ongoing. Composite CdTe(211)B/GaAs(211)B substrates have recently attracted renewed attention because of the ready availability of large-area $\operatorname{GaAs}(211) \mathrm{B}$ wafers as well as the ease of their surface preparation relative to $\mathrm{Si}$ substrates due to the easier in situ

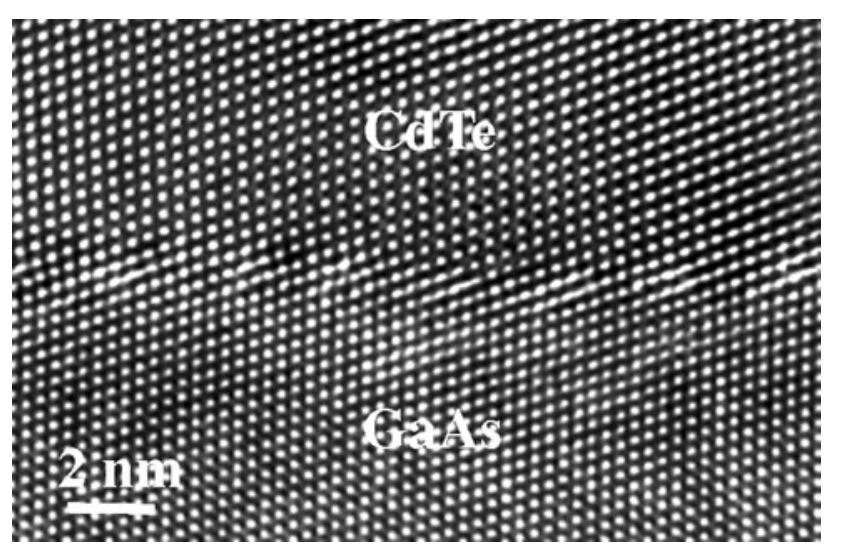

Fig. 7. High-resolution electron micrograph of $\mathrm{CdTe}(211) /$ $\operatorname{GaAs}(211)$ interface. Note $\sim 2.6^{\circ}$ rotation between vertical $\{111\}$ lattice fringes. ${ }^{25}$

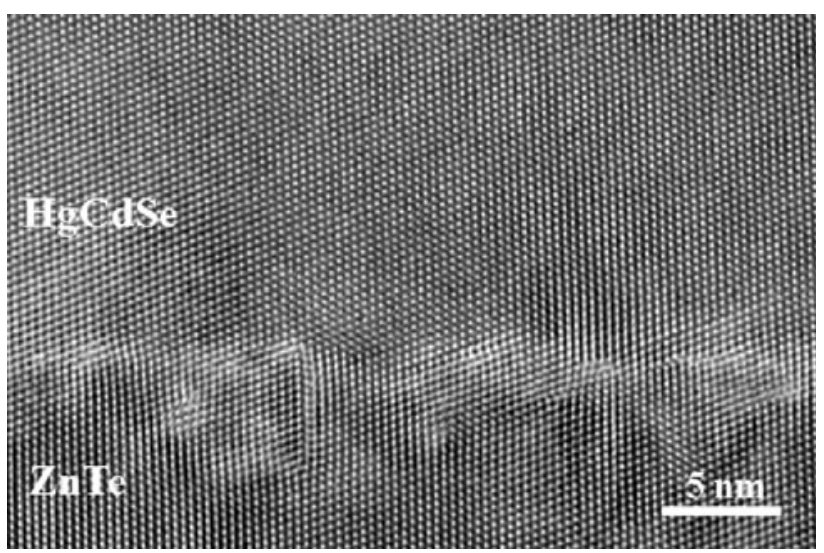

Fig. 8. High-resolution electron micrograph of $\mathrm{HgCdSe}(211) / \mathrm{ZnTe}$ heterointerface from sample grown at $100^{\circ} \mathrm{C}$ on $\mathrm{ZnTe} / \mathrm{Si}(211) \mathrm{com}$ posite substrate. ${ }^{28}$

surface oxide removal. ${ }^{2}$ Several cycles of annealing were used during deposition of the thick CdTe buffer layers in efforts to reduce the overall density of 


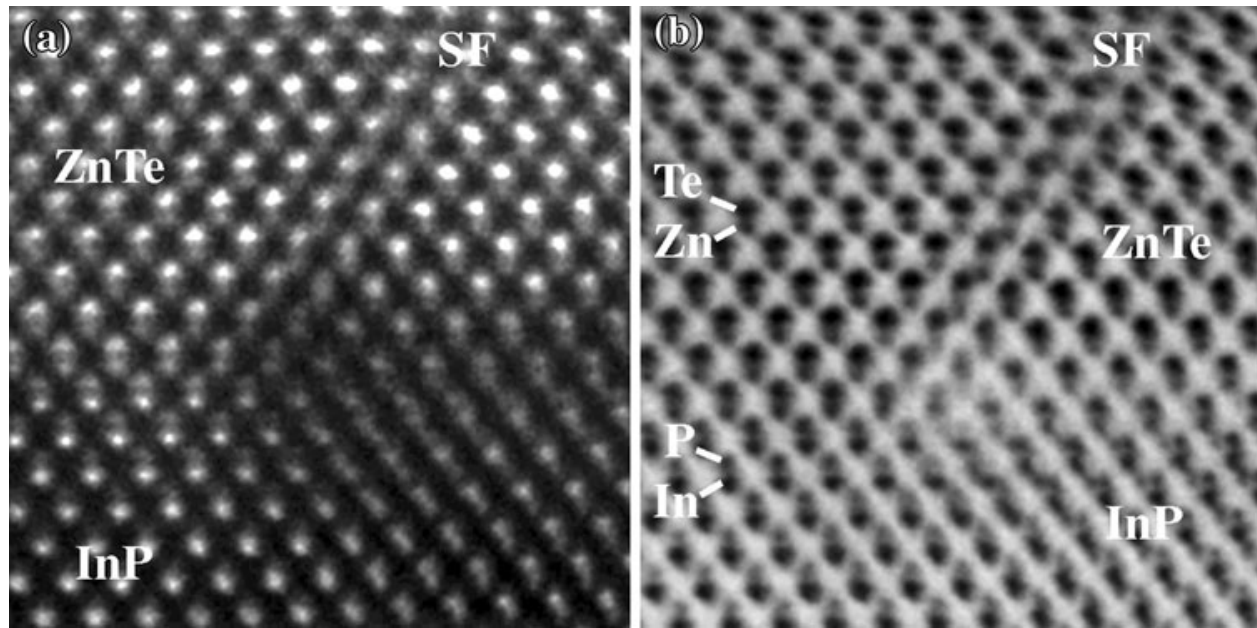

Fig. 9. Aberration-corrected electron micrographs showing \{111\}-type stacking fault (SF) originating at ZnTe(100)/InP(100) interface: (a) HAADF scanning TEM (STEM) image (collection angle of $\sim 90 \mathrm{mrad}$ to $170 \mathrm{mrad}$ ), (b) BF STEM image (collection angle $\sim 22 \mathrm{mad}$ ).

threading dislocations, and TEM cross-section observations confirmed that a large decrease had occurred upon moving away from the region of the $\mathrm{CdTe} / \mathrm{GaAs}$ interface. ${ }^{25}$ Figure 7 shows a high-resolution electron micrograph demonstrating the abruptness of the $\mathrm{CdTe}(211) / \mathrm{GaAs}(211)$ interface: the rotation angle between the vertical $\{111\}$-type lattice fringes of the CdTe epilayer and the GaAs substrate was determined to be $\sim 2.6^{\circ}$ for these two materials. Fourier filtering was also applied to highresolution lattice images from the interfacial region, and analysis of the residual strain indicated that the interface was effectively fully relaxed except for some locations where polishing had left small pits in the GaAs substrate surface. ${ }^{25}$

\section{HgCdSe}

\section{ZnTe/Si(211) and GaSb(211) Substrates}

HgSe crystallizes in the zincblende structure, as does CdSe when grown by molecular beam epitaxy. ${ }^{26}$ Thus, $\mathrm{Hg}_{1-x} \mathrm{Cd}_{x} \mathrm{Se}$ alloys could in principle be grown with adjustable band gaps that depend on composition, similar to the case of $\mathrm{Hg}_{1-x} \mathrm{Cd}_{x} \mathrm{Te}$. Moreover, HgCdSe alloys should be nearly latticematched with InAs or GaSb, which could then be used to provide suitable large-area substrates. Thus, the prospect of IR detectors based on HgCdSe has stimulated renewed efforts to grow epitaxial HgCdSe films. ${ }^{27}$ Early growth experiments established that the temperature range for optimal $\mathrm{HgCdSe}$ was restricted to between $80^{\circ} \mathrm{C}$ and $100^{\circ} \mathrm{C}$. Later TEM observations revealed greatly increased defect density for films grown at higher temperature. ${ }^{28}$ The high-resolution electron micrograph in Fig. 8 shows the interface region for a thin $\mathrm{Hg}_{0.71} \mathrm{Cd}_{0.29} \mathrm{Se}$ film grown at $100^{\circ} \mathrm{C}$ on a $\mathrm{ZnTe} / \mathrm{Si}$ composite substrate; the high quality of the epitaxial growth is clearly evident. $\mathrm{HgCdSe}$ films have also been deposited directly onto $\mathrm{GaSb}(211)$ substrates, but the quality of the epilayers grown was compromised somewhat by the relative lack of flatness of the GaSb(211) substrate surface, so further work is still required to develop a better surface etching or polishing protocol.

\section{Aberration-Corrected Electron Microscopy}

The emergence of techniques for correction of third-order spherical aberration, once considered as an insurmountable resolution-limiting defect of round magnetic electron lenses, has had a profound impact on the microscopy and materials communities. $^{29}$ Microscope information limits have been extended to better than $1 \AA$, in particular making it possible to resolve projections of individual atomic columns in additional low-index zone-axis orientations of elemental and compound semiconductors. Several viable approaches to ACEMs have been demonstrated: off-axis electron holography and focal-series reconstruction use offline software to achieve aberration correction, while in-column hardware correctors have been developed for both conventional transmission (image-corrected) and scanning (probe-corrected) instruments. ${ }^{28}$ Results reported in the literature so far seem to heavily favor the probe-corrected approach, which incidentally provides several different imaging geometries. ${ }^{8}$ These include: high-angle annular dark-field (HAADF) or Z-contrast imaging, which has historically been used in scanning TEM; bright-field (BF) imaging, which is much less noise-limited in a probe-corrected instrument; and annular BF, which involves an annular detector placed within the incident cone of illumination. ${ }^{8}$ Figure 9 compares aberration-corrected HAADF and $\mathrm{BF}$ images of an inclined $\{111\}$-type stacking fault originating at a $\mathrm{ZnTe} / \mathrm{InP}$ heterointerface. The atomic "dumbbells" of the two materials are clearly visible in each case, and it is also straightforward to recognize the 
stacking of the individual $\mathrm{Zn}$ and Te atomic columns across the defect. Although optimization of imaging conditions for studying these and similar compound semiconductors has not yet been settled, these two micrographs clearly demonstrate the enormous potential of ACEM imaging for determining atomic configurations at defects and interfaces.

\section{CONCLUSIONS}

Atomic resolution with the electron microscope has been extensively used to characterize the microstructure of II-VI zincblende semiconductors grown epitaxially on a variety of commonly used substrates. Composite substrates are also being employed to overcome lattice-mismatch problems before final growth of the zincblende material of primary interest. Work continues on substrate surface preparation and optimization of deposition conditions, again necessitating close collaboration between electron microscopists and crystal growers to ensure that materials of the highest possible quality are reliably grown. Meanwhile, much more work still remains to be done to exploit the potential of ACEMs for examining structural defects in these and related materials at unprecedented resolution levels.

\section{ACKNOWLEDGEMENTS}

The recent work at Arizona State University has been supported by the US Army Research Laboratory and the US Army Research Office under Contract \#54657EL (Monitor: Dr. W.W. Clark). Ongoing collaborations with the groups of J.K. Furdyna, X. Liu (Notre Dame), R.N. Jacobs (NVESD), Y. Chen, G. Brill (ARL), and Y.H. Zhang (Arizona State University), as well as contributions to this work by former graduate students T. Aoki, J.J. Kim, L. Ouyang, X. Zhang, and W.F. Zhao, are gratefully acknowledged. The use of facilities in the John M. Cowley Center for High Resolution Electron Microscopy is also acknowledged. Acquisition of the JEOL-ARM200F at Arizona State University was supported by NSF Grant DMR-0820196.

\section{REFERENCES}

1. M.A. Kinch, J. Electron. Mater. 39, 1043 (2010).

2. R.N. Jacobs, C. Nozaki, L.A. Almeida, M. Jaime-Vasquez, C. Lennon, J.K. Markunas, D. Benson, P. Smith, W.F. Zhao, D.J. Smith, C. Billman, J. Arias, and J. Pellegrino, J. Electron. Mater. 41, 2707 (2012).
3. Y. Chen, S. Simingalam, G. Brill, P. Wijewarnasuriya, N. Dhar, J.J. Kim, and D.J. Smith, J. Electron. Mater. 41, 2917 (2012).

4. D.J. Smith, Rep. Prog. Phys. 60, 1513 (1997).

5. R.W. Glaisher, A.E.C. Spargo, and D.J. Smith, Ultramicroscopy 27, 131 (1989).

6. M.A. O'Keefe, L.F. Allard, and D.A. Blom, J. Electron Microsc. 54, 169 (2005).

7. F. Phillipp, Adv. Solid State Phys. 35, 257 (1996).

8. D.J. Smith, T. Aoki, J. Mardinly, L. Zhou, and M.R. McCartney, Microscopy 62, S65 (2013).

9. T. Aoki, Y. Chang, G. Badano, J. Zhao, C. Grein, S. Sivananthan, and D.J. Smith, J. Cryst. Growth 265, 224 (2004).

10. A.G. Cullis, N.G. Chew, and J.L. Hutchison, Ultramicroscopy 17, 203 (1985).

11. C. Wang, D.J. Smith, S. Tobin, T. Parados, J. Zhao, Y. Chang, and S. Sivananthan, J. Vac. Sci. Technol. A 24, 995 (2006).

12. J. Fan, L. Ouyang, X. Liu, D. Ding, J.K. Furdyna, D.J. Smith, and Y.-H. Zhang, J. Cryst. Growth 323, 127 (2011).

13. L. Ouyang, J. Fan, S. Wang, X. Lu, Y.-H. Zhang, X. Liu, J.K. Furdyna, and D.J. Smith, J. Cryst. Growth 330, 30 (2011).

14. X. Zhang, S. Wang, D. Ding, X. Liu, J.-H. Tan, J.K. Furdyna, Y.-H. Zhang, and D.J. Smith, J. Electron. Mater. 38, 1558 (2009).

15. D.J. Smith, S.-C.Y. Tsen, D. Chandrasekhar, P.A. Crozier, S. Rujirawat, G. Brill, Y.P. Chen, R. Sporken, and S. Sivananthan, Mater. Sci. Eng. B 77, 93 (2000).

16. L.A. Almeida, Y.P. Chen, J.P. Faurie, S. Sivananthan, D.J. Smith, and S.-C.Y. Tsen, J. Electron. Mater. 25, 1402 (1996).

17. D.J. Smith, S.-C.Y. Tsen, Y.P. Chen, S. Sivananthan, and J.B. Posthill, Appl. Phys. Lett. 69, 2086 (1996).

18. S. Rujirawat, Y. Xin, N.D. Browning, S. Sivananthan, D.J. Smith, S.-C.Y. Tsen, Y.P. Chen, and Y. Nathan, Appl. Phys. Lett. 74, 2346 (1999).

19. S. Rujirawat, D.J. Smith, J.P. Faurie, G. Neu, V. Nathan, and S. Sivananthan, J. Electron. Mater. 27, 1047 (1998).

20. R.N. Jacobs, J. Markunas, J. Pellegrino, L.A. Almeida, M. Groenert, M. Jaime-Vasquez, N. Mahadik, C. Andrews, and S.B. Qadri, J. Cryst. Growth 310, 2960 (2008).

21. W.F. Zhao, R.N. Jacobs, M. Jaime-Vasquez, L.O. Bubulac, and D.J. Smith, J. Electron. Mater. 40, 1733 (2011).

22. J.-J. Li, X. Liu, S. Liu, S. Wang, D.J. Smith, D. Ding, S.R. Johnson, J.K. Furdyna, and Y.-H. Zhang, Appl. Phys. Lett. 100,121908 (2012).

23. T. Aoki, D.J. Smith, Y. Chang, J. Zhao, G. Badano, C. Grein, and S. Sivananthan, Appl. Phys. Lett. 82, 2275 (2003).

24. T. Aoki, M. Takeguchi, P. Boieriu, R. Singh, C. Grein, Y. Chang, S. Sivananthan, and D.J. Smith, J. Cryst. Growth 271, 29 (2004).

25. J.J. Kim, R.N. Jacobs, L.A. Almeida, M. Jaime-Vasquez, C. Nozaki, and D.J. Smith, J. Electron. Mater. (2013). doi: 10.1007/s11664-013-2688-7.

26. N. Samarth, H. Luo, J.K. Furdyna, S.B. Qadri, Y.R. Lee, A.K. Ramdas, and N. Otsuka, Appl. Phys. Lett. 54, 2680 (1989).

27. G. Brill, Y. Chen, and P. Wijewarnasuriya, J. Electron. Mater. 40, 1679 (2011).

28. W.F. Zhao, G. Brill, Y. Chen, and D.J. Smith, J. Electron. Mater. 41, 2852 (2012).

29. D.J. Smith, Microsc. Microanal. 14, 2 (2008). 